\title{
Design and Test of a High QoS Radio Network for CBTC Systems in Subway Tunnels
}

\author{
C. Cortés Alcalá* , Siyu Lin**, Ruisi He** C. Briso-Rodriguez* \\ *EUIT Telecomunicación. Universidad Politécnica de Madrid, \\ 28031, Madrid Spain. \\ **State Key LAboratory of Rail Traffic Control and Safety. \\ Beijing Jiaotong University,100044, Beijing, China. \\ E-mail Cbriso@diac.upm.es
}

\begin{abstract}
Communications Based Train Control Systems require high quality radio data communications for train signaling and control. Actually most of these systems use $2.4 \mathrm{GHz}$ band with proprietary radio transceivers and leaky feeder as distribution system. All them demand a high QoS radio network to improve the efficiency of railway networks. We present narrow band, broad band and data correlated measurements taken in Madrid underground with a transmission system at $2.4 \mathrm{GHz}$ in a test network of $2 \mathrm{~km}$ length in subway tunnels. The architecture proposed has a strong overlap in between cells to improve reliability and QoS. The radio planning of the network is carefully described and modeled with narrow band and broadband measurements and statistics. The result is a network with $\mathbf{9 9 . 7 \%}$ of packets transmitted correctly and average propagation delay of $20 \mathrm{~ms}$. These results fulfill the specifications QoS of CBTC systems.
\end{abstract}

Index Terms - Railway, CBTC, train control, Tunnels, quality of service, simulcast.

\section{INTRODUCTION}

Railways are becoming very important for mass people movement. Advanced metropolitan railway must have high capacity, reliability and security and these requires the use of communications based train control systems (CBTC) [1],[2]. This technology is basic for train capacity and is beginning to get used in most advanced subways.

CBTC is an automated control system for railways that improves and ensures the safe operation of rail vehicles using wireless data communication between the trains and the control centre. CBTC is a modern successor of the traditional railway signaling systems which provide a limited control using track circuits, interlocking, and signals.

All CBTC systems operate in the same basic way: an off board computer can control all trains operating on a track linked to the computer. This allows trains to be spaced closer together than on a manually controlled line. The computer sends information to the computers aboard the train using wireless communications.

CBTC systems use a radio data communications network to send objective speed information to the trains and to receive location and actual speed from trains. The system uses a communications network composed of several base stations connected to the train control system. The off board computer is polling one by one all the moving train in a part of the track.
Each train polled must answer sending their location and speed and then the off board computer sends the objective speed or distance to each train according to their relative position. This information is updated every 100 to $300 \mathrm{~ms}$. If one train do not answer during a period of time from $500 \mathrm{~ms}$ to 1 second all trains in the control area must reduce speed or carry out and emergency braking. Therefore radio network must have a high quality of service and an low transmission delay.

Most of modern CBTC systems use data communications in the $2.4 \mathrm{GHz}$ band with proprietary radio transceivers [3]. To achieve a high quality of service most of new networks are using leaky feeder [4] as transmission system in between radio base stations and trains.

Leaky feeder network in a complex tunnels environment of a large subway is difficult and expensive to install and do not guarantee a high QoS in all cases. Propagation studies [5] [6] have shown that radio coverage in tunnels can also be achieve with good quality using antennas. We proposed a solution based on the use of base stations with antennas. This solution requires more engineering work but it is fast and easy to deploy than a leaky feeder and our test has shown that it can reach a high quality of service in a real complex environment of tunnels. The antennas solution has a cost 1/4 of leaky feeder solution and also installation and maintenance is easier and cheaper.

On this paper we present the complete test of a radio network with high QoS for broadband data transmissions in subway tunnels. The solutions has been modeled and tested with narrow band and broadband measurements, and data transmissions. All the results are correlated so we can easy and carefully evaluate the influence of propagation in the transmission data errors.

\section{MEASUREMENTS SYSTEM AND ENVIRONMENT}

The objective of the measurements was to evaluate the quality of service obtained with a wireless transmission system in a complex tunnels environment at $2.4 \mathrm{GHz}$ using antennas. For this purpose three types of measurements were made: Narrow band propagation, broadband channel modeling and data transmission measurements. The results are correlated and can be used to evaluate quality of service (QoS) of the channel and two verify the good design of the radio network.

The measurements have been carried out in a typical line of Madrids underground. Fig. 1 shows the section of the tunnels, 
where a $2000 \mathrm{~m}$ length track has been selected for measuring with one station at the beging, one in the middle an one at the end, and the transmitters separated $400 \mathrm{~m}$ in between them The stations were separated $400 \mathrm{~m}$ one from each other, according to propagation computation [5].

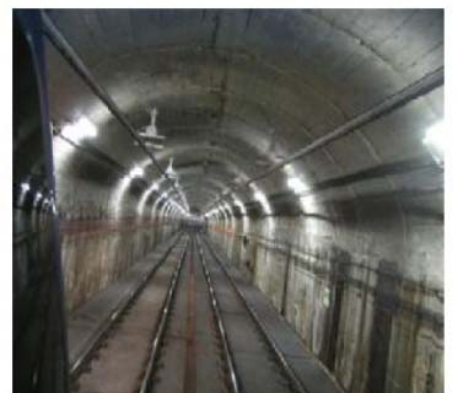

(a)

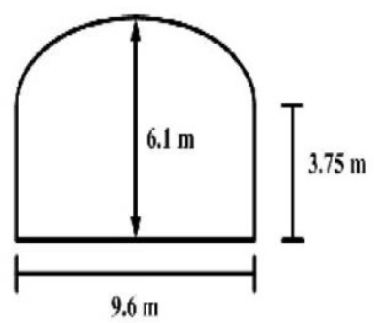

(b)
Fig.1. Typical tunnel of the test track. Arched shape with $6.1 \mathrm{~m}$ maximum height in the middle and $9.6 \mathrm{~m}$ width

\section{A. Narrow band measurements}

The test system for narrow band measurements was composed of four $20 \mathrm{dBm}$ transmitters, each one with two $12 \mathrm{dBi}$ antennas, circular polarization. Each antenna was pointing to one side of the tunnel. Inside the tunnel, the antennas were located $4 \mathrm{~m}$ over the floor and $25 \mathrm{~cm}$ from the tunnel walls, as shown in Fig. 4.

The receiver was installed on a $60 \mathrm{~m}$ length standard train, with two antennas, one in the front and one in the rear.

The antennas of the train were $8 \mathrm{dBi}$ gain, linear polarization and each one have a low noise amplifier attached. The output o both antennas were combined with a power splitters at the input of the receiver. The receiver was a sprectum analyzer controlled with a PC computer and a special software. Position was recorded from train tachometer.

The test system was able to take 100 measurements per second. With this recording speed we have got a rate of one measurement each $1 / 2$ wavelength at $2.4 \mathrm{GHz}$ with a maximum train speed of $20 \mathrm{~km} / \mathrm{h}$.

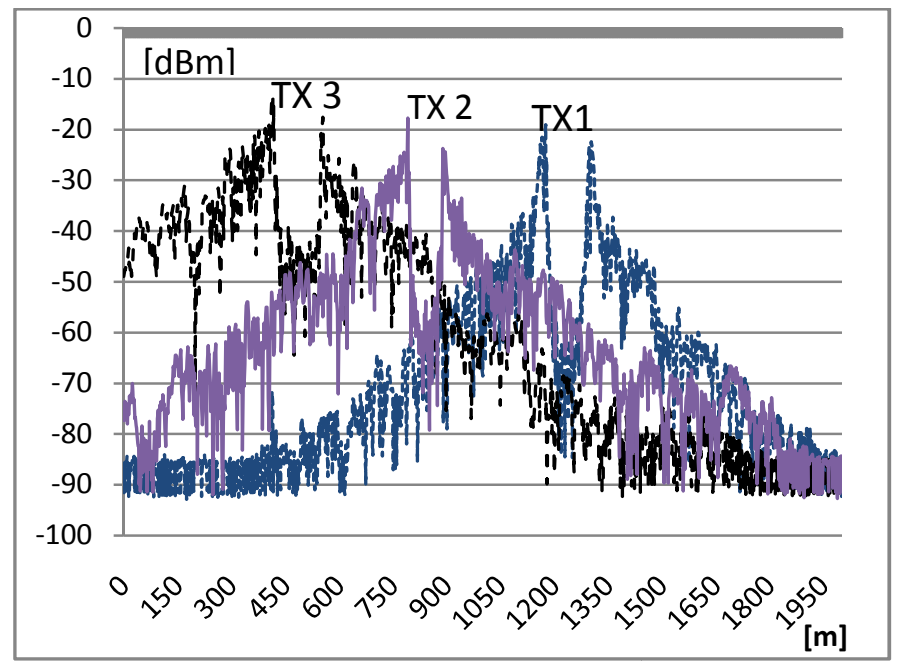

Fig.2. Power along the track. Narrow band transmission.
The first results, Fig. 2, are narrow band measurements of the coverage of the three transmitters. We can see that the coverage is very good except by a strong fading right when the train pass in front of the transmitter. On this case there are no line of sight in between antennas of the train and transmitters.

The rest of the time the signal level is over $-60 \mathrm{dBm}$ along $2000 \mathrm{~m}$ the track.

Basic statistics from point 300 to 2300 and best fit statistical fucntions for fast fading and slow fading are shown in Table I. This parameters are simple and easy to measure and can be used for basic radio planning.

TABLE I

NARROW BAND STATISTICS

\begin{tabular}{lc}
\hline \hline \multicolumn{1}{c}{ Parameter } & VALUE \\
\hline Average signal power & $-54.2 \mathrm{dBm}$ \\
Standard deviation & $11 \mathrm{~dB}$ \\
Fast fading & Rice $/ \mathrm{K}=5.2(7.16 \mathrm{~dB})$ \\
Slow fading & Log normal $/ \sigma=3.8 \mathrm{~dB}$
\end{tabular}

\section{B. Broadband measurements}

The second group of measurements was made using a channel sounder installed in the position of TX3 with the same antenna configuration. This sounder was used for special modelling of the behaviour of propagation close to the transmitter.

The sounder uses the frequency domain technique transmitting a flat $5 \mathrm{MHz}$ spectrum at $2400 \mathrm{MHz}$ and recording the response of the channel with a mobile receiver.

Results are shown on Fig.3 with 3D graph of distance, bandwidth and signal power received. On the graph we have $n$ distinguish three propagation regions: Line of Sight (LOS), Non Line of Sight (NLOS) and far line of sight (FLOS). This three regions have different behavior and multipath conditions. Detailed 6 ray delay and amplitude model has been obtained from measurements and is given on Table II.

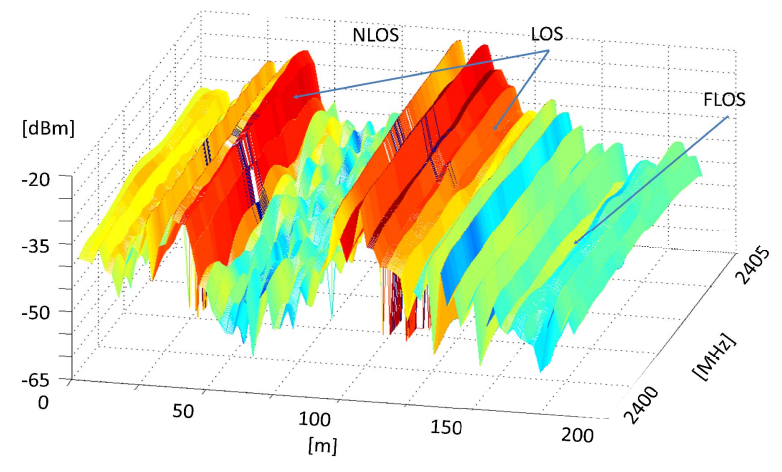

Fig. 3. Measured 3D wideband received signal along 200m track. 5Mhz bandwidth at $2.4 \mathrm{GHz}$.

Interesting results are the long propagation delays along the tunnel due to reflections in discontinuities like stations or curves that spread the RMS delay to $250 \mathrm{~ns}$ in NLOS conditions. 
This situation limits the coherence bandwidth from $100 \%$ (5Mhz) in LOS to $20 \%$ in NLOS region limiting communications on this situation.

Also far from the transmitter (FLOS) there is a small coherence bandwidth reduction that can reduce data bandwidth and increase error probability.

TABLE II

BROADBAND MODEL

\begin{tabular}{cc|cc|cc}
\hline \multicolumn{2}{c|}{ LOS } & \multicolumn{2}{c|}{ NLOS } & \multicolumn{2}{c}{ FLOS } \\
\hline $\begin{array}{c}\text { Delay } \\
(\mathrm{ns})\end{array}$ & $\begin{array}{c}\text { Amplitude } \\
(\mathrm{dB})\end{array}$ & $\begin{array}{c}\text { Delay } \\
(\mathrm{ns})\end{array}$ & $\begin{array}{c}\text { Amplitude } \\
(\mathrm{dB})\end{array}$ & $\begin{array}{c}\text { Delay } \\
(\mathrm{ns})\end{array}$ & $\begin{array}{c}\text { Amplitude } \\
(\mathrm{dB})\end{array}$ \\
\hline 0 & 0 & 0 & 0 & 0 & 0 \\
200 & 30.68 & 200 & 16.13 & 400 & 27.95 \\
400 & 34.29 & 600 & 32.9 & 600 & 30.2 \\
600 & 28.49 & 800 & 30.02 & 900 & 36.56 \\
1000 & 31.99 & 1200 & 13.76 & 1200 & 30.44 \\
1400 & 40.35 & 1600 & 24.82 & 1400 & 24.76 \\
\hline
\end{tabular}

RMS Delay Spread

\begin{tabular}{c|c|c}
\hline $37.5 \mathrm{~ns}$ & $250.6 \mathrm{~ns}$ & $92.3 \mathrm{~ns}$ \\
\hline \multicolumn{3}{|c}{ Coherence Bandwidth } \\
\hline $5 \mathrm{MHz}$ & $0.8 \mathrm{MHz}$ & $2.2 \mathrm{MHz}$ \\
\hline
\end{tabular}

\section{DATA TRANSMISSION}

The second part of the test want to evaluate the effect of the radio coverage on the transmission network and the efficiency of the strong overlap of radio coverage. For this purpose measurements where made using data transmissions to verify the behavior of the channel in real conditions.

The test network has three base stations of the type of Fig. 4, each one with a PC computer and $2.4 \mathrm{GHz}$ CDMA radio [7] transmitting a $250 \mathrm{Kbps}$. The antennas used were the same of the other cases: two $12 \mathrm{dBi}$ directional antenna, and the transmission power was the same $20 \mathrm{dBm}$.

The train has two antennas and two radios, one in the front and one in the rear. Both radios are connected to a test computer as shown in Fig. 5. The test computer can collect data packets from the front or rear antenna. The characteristics of the radio and antennas are given in Tab. III.

Each base sends a data packets to the train every $150 \mathrm{~ms}$. The packets have the number of the base station, packet number and a time reference used for delay measurements with $1 \mathrm{~ms}$ resolution. The three base stations use the same radio channel.

The measurement equipments were deployed in the locomotive cabin of one of the trains. The trains used were standard Madrid subway trains with four cars and $60 \mathrm{~m}$ length..

The mobile station was polling each one of the three base stations every $150 \mathrm{~ms}$ and was receiving the answer packet from the base stations through the on board radios. The PC receives the data packets and can identify the origin, packet lost and delay. There are no erroneous packets received by the computer because the radios can correct some errors in the packets or if it can't be corrected then it is considered lost. Also the data transmission protocol is User Datagram Protocol UDP, so that it is not connection oriented and there are no acknowledge of the transmission.

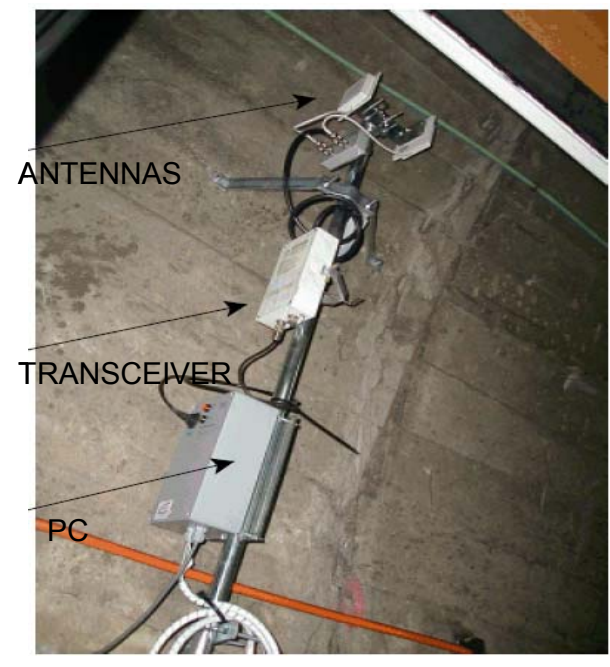

Fig. 4.. Data test system with CDMA radio.

On a real network there will be only one main computer and it will be connected with the base stations through a local area network. This computer will control the trains in a region polling each train with a data packet transmitted through the fixed network and each train will answer when polled.

The polling packets are composed of the 1024 bits.

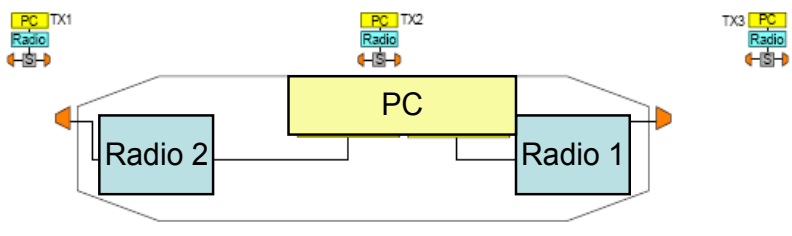

Fig. 5. On board equipment and radio network.

TABLE III

CHARACTERISTICS OF RADIO SYSTEM

\begin{tabular}{lc}
\hline \hline \multicolumn{1}{c}{ Characteristic } & VALUE \\
\hline Frequency & $2,400-2,4835 \mathrm{GHz}$ \\
Radio type & DSSS (direct Sequence Spread Spectrum) \\
Transmission power & $0-23 \mathrm{dBm}$ \\
Transmission bandwidth & $4,6 \mathrm{MHz}$ \\
Data rate & $1 \mathrm{Mbps}$ \\
Rx sensitivity & $-90 \mathrm{dBm} @ 1 \mathrm{Mbps}$ \\
Antennas: base station & Two 12 dBi circular polarization \\
Antennas mobile station & $12 \mathrm{dBi}$ vertical linear polarization
\end{tabular}

This test configuration was been used to make data transmissions trials along the track for each transmitter and general results are shown in Fig 6, for the three TX and for the complete network. These results has been correlated with the narrow band and broadband propagation modeling made in the tunnel, to obtain an specification of signal power and quality for a radio network for CBTC systems in tunnels. 

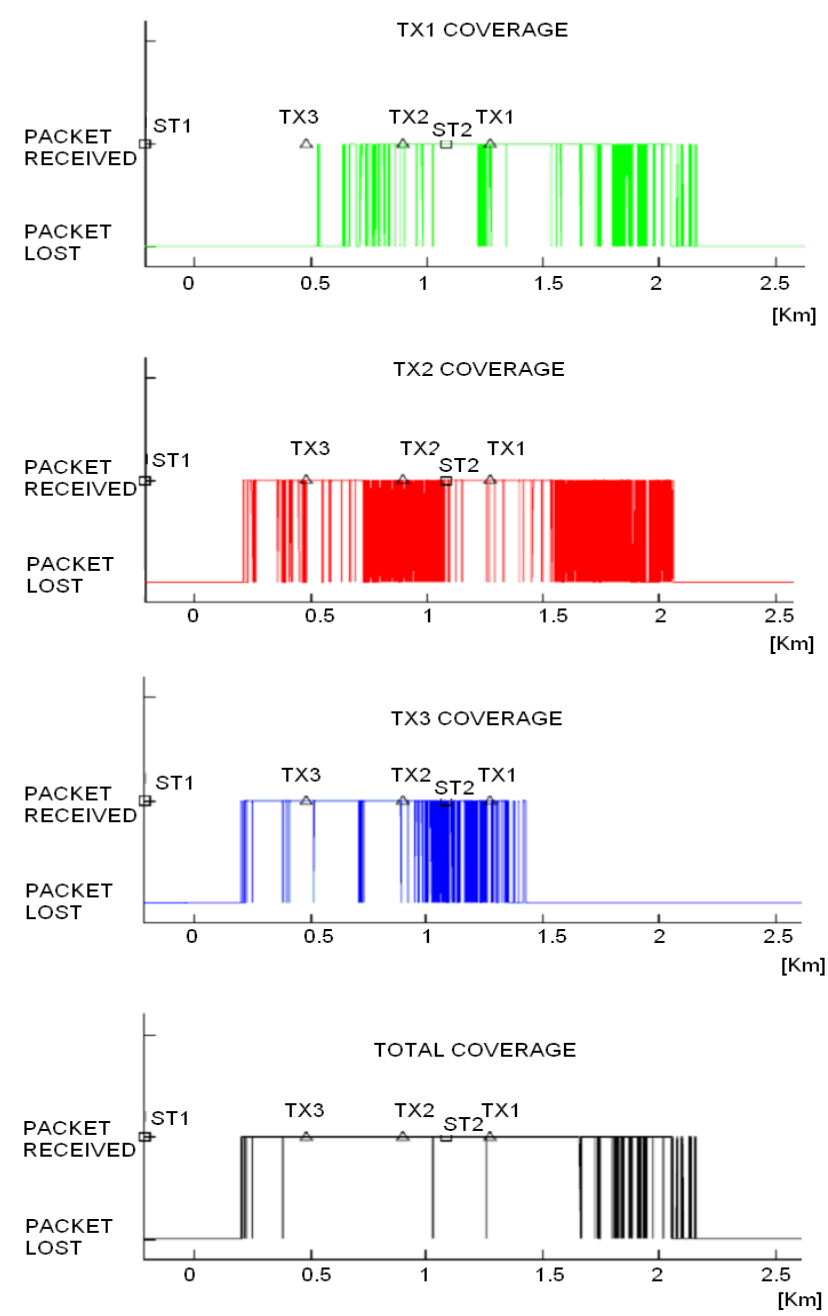

Fig. 6. Packets received by the mobile station from each transmitter and the total coverage. Train with two radios one in the front and one in the rear.

On Fig. 6 we can see that the radio coverage of each transmitter is up to $1.5 \mathrm{Km}$ according to the sensitivity of the receiver that is $-90 \mathrm{dBm}$ and signal power of measurements of Fig. 2.

The QoS for a single transmitter is poor with areas with very bad quality when the train pass in front of the transmitter, or in the region in between two transmitters

The statistics for each single transmitters are shown on Table IV, where we can see that the average packet lost is very high, from $14 \%$ to $27 \%$. These results are unacceptable for CBTC systems.

Fig. 6 also shows the total coverage of the network. This graph represents the recovered packets along the complete test track. We can see on the figure and on Table $\mathrm{V}$ that the result of total coverage is fairly good with an average packet lost of $0.7 \%$ (Tab. V). These results are obtained because the train is collecting packets from the front and rear radios and as there is a strong overlap of the cells, there is always a good packet received.

The solution also solves the problem of NLOS propagation in front of the transmitters due to self shadowing of the train. Therefore the architecture of the system with three cells with strong signal overlap has result very reliable for data transmissions and are fully applicable to CBTC systems.
TABLE IV

Statistics OF THE COVERAGE OF EACH TRANSMITTER

\begin{tabular}{|c|c|c|c|c|c|c|}
\hline \multirow{2}{*}{ Packets } & \multicolumn{2}{|c|}{ TX1 } & \multicolumn{2}{|c|}{ TX2 } & \multicolumn{2}{|c|}{$\mathrm{TX} 3$} \\
\hline & VALUE & $\%$ & VALUE & $\%$ & VALUE & $\%$ \\
\hline Transmitted & 3601 & 100 & 1653 & 100 & 2038 & 100 \\
\hline Received & 2623 & 72,8 & 1419 & 85,8 & 1643 & 78,9 \\
\hline Lost & 978 & 27,2 & 234 & 14,2 & 440 & 21,1 \\
\hline
\end{tabular}

TABLE V

STATISTICS OF THE TOTAL COVERAGE

\begin{tabular}{lcc}
\hline & VALUE & $\%$ \\
& & \\
\hline Transmitted packets & 2.017 & 100 \\
Received packets & 2011 & 99.70 \\
Error packets & 0 & 0 \\
Lost packets & 6 & 0,30
\end{tabular}

\section{CONCLUSION}

The use of antenna solutions for subway tunnels at $2.4 \mathrm{Ghz}$ has been widely study with accurate measurements of signal power, coherence bandwidth and data transmissions.

We have measured and characterized problems when the train pass close to the TX, the fast fading and multipath propagation close and far from the transmitter. With these results a network architecture with strong overlap of radio coverage overlap in between neighbour cells has been proposed and tested.

The network design adds diversity to the reception of the data on the trains and combined with simultaneous transmissions of the same packet from all TX (simulcast) can be used for CBTC system reaching efficiency of transmission higher than $99 \%$ of packets transmitted.

The solution can efficiently replace leaky feeder transmission networks with improvements in quality and specially in deployment cost.

\section{ACKNOWLEDGMENT}

We also would like to thank to the Madrid Subway Carrier (METRO) and to Dimetronic Invensys for their help and support to perform the propagation measurements.

\section{REFERENCES}

[1] "IEEE standard for Communications-Based Train Control (CBTC) performance and functional requirements," 30. Dec. 1999.

[2] Alexander, R.P.; Mortlock, E.A.; Hamilton, B.A "Controlling and executing communications based train control (CBTC) installation \& testing with a CBTC- ready vehicle." Rail Conference, 2005. Proceedings of the 2005 ASME/IEEE Joint. 2005, pp.193 - 198

[3] Fitzmaurice, M. "Use of $2.4 \mathrm{GHz}$ frequency band for Communications Based Train Control data communications systems". Rail Conference, 2006. Proceedings of the 2006 IEEE/ASME Joint. 2006,pp:263 - 267

[4] R. J. Jakubowski, "Results of distributed antenna and leaky feeder systems tests at $800 \mathrm{MHz}$ in Washington D. C. metro system tunnels," IEEE 44th Veh. Technol. Conf., vol. 2, 1994, pp. 1113-1116. 
[5] C. Briso-Rodriguez, J.M. Cruz and J.I. Alonso, " Measurements and Modeling of Distributed Antenna Systems in Railway Tunnels," Veh. Technol., IEEE Trans. vol. 56, Issue 5, Part 2, pp.2870 - 2879, Sept. 2007.

[6] M. Lienard and P. Degauque, "Propagation in wide tunnels at $2 \mathrm{GHz}$ : A statistical analysis," IEEE Trans. Veh. Technol., vol. 47, no. 4, pp. 1322 1328, Nov. 1998.

[7] Yuan Cao; Ru Niu; Tianhua Xu; Tao Tang; Jiancheng Mu; "Wireless test platform of Communication Based Train Control (CBTC) system in urban mass transit. "Vehicular Electronics and Safety, 2007. ICVES. IEEE International Conference on 2007 pp:1 - 4 .

[8] Li Zhu; Yan Zhang; Bin Ning; Hailin Jiang;Train-Ground Communication in CBTC Based on 802.11b: Design and Performance Research. Communications and Mobile Computing, 2009. CMC '09. WRI International Conference Vol. 2, 2009, pp:368 - 37. 